\title{
LA DEMANDA DE LOS PERIÓDICOS IMPRESOS Y ONLINE: UN ANÁLISIS ECONÓMICO
}

\author{
Julio Larrañaga \\ (Universidad Complutense de Madrid) \\ juliolr03@hotmail.com
}

\begin{abstract}
Resumen: Se está produciendo un cambio en las pautas de consumo de los periódicos impresos, con una continúa reducción del número de lectores más jóvenes, por otra parte las nuevas tecnologías están acelerando este proceso de cambio. Los jóvenes acuden a Internet en su demanda de noticias, y es además cada vez mayor el público de otras edades y de distintas clases sociales que también acude a la Red con este mismo objetivo. En Internet los periódicos online no sólo tienen que competir con las web de los otros medios, sino también con otros proveedores online de noticias.
\end{abstract}

Palabras clave: Periódicos online, Internet, prensa, audiencias.

Abstract: A change is taking place in the consumption patterns of printed newspapers, to the extent that a steady decline of customers is the trend, especially as regards the younger strata, and the widespread use of new technologies acts as a reinforcing factor in these changing habits. Young people use internet in order to supply their news demand, and this is also the means that other age groups and people from different social strata are taking advantage of. In Internet newspapers on-line must not only compete with the web-sites of other news media but also with other news providers.

Keywords: Online newspapers, Internet, press, audiences.

\section{INTRODUCCION}

n los últimos años y en diferentes países se está constatando un cambio en el consumo de los periódicos impresos. Además, las nuevas tecnologías están acelerando este proceso. A lo largo de este artículo se analizan desde el punto de vista económico las principales características de los lectores de los periódicos impresos y de los periódicos online en España. La exposición se completa con el análisis de las principales conductas de los internautas españoles.

\section{LA DEMANDA DE NOTICIAS EN INTERNET}

Un porcentaje muy elevado de internautas españoles utiliza Internet como fuente de información. Según los datos que proporciona el informe de la Fundación BBVA "Segundo Estudio sobre Internet en España", el 82,0\% de ellos busca información en Internet, y el $46,7 \%$ lee noticias o periódicos online. Por otra parte, los internautas que utilizan Internet con más frecuencia estiman que 
24 La demanda de los periódicos impresos y online: un análisis económico

la información que esperan encontrar allí es fiable y correcta. Si se realiza una valoración de 0 a 10, y se asigna como 0 a la situación en que no se espera en absoluto encontrar información fiable y correcta, y como valor 10 a la situación en que espera encontrar la información de forma completa, los internautas encuestados por la Fundación BBVA asignan elevados valores a la información disponible en Internet de diferentes temas. Concretamente a la referida a salud, le asignan un valor de 7,2 y a ciencia y tecnología también 7,2. Mientras tanto asignan puntuaciones menores a las informaciones disponibles sobre economía con 6,6 y política con 6,2. Por lo tanto, en España el grado de confianza de los internautas en la información de la Red es muy elevado.

Resultados parecidos se pueden obtener en otros informes sobre Internet en nuestro país, por ejemplo en la " $10^{\mathrm{a}}$ encuesta AIMC a usuarios de Internet" que realiza la Asociación para la Investigación de Medios de Comunicación (AIMC). En ella, el $49,8 \%$ de los encuestados indican que Internet es su fuente fundamental de noticias para estar informados sobre la actualidad, y para el $43,8 \%$ de ellos si bien es una fuente secundaria, es importante. Además se especifica en dicha encuesta que la lectura de periódicos online es una costumbre que está muy consolidada en los internautas españoles, pues el $67,2 \%$ de los encuestados han afirmado que el día anterior a la realización de la encuesta de la AIMC, han leído un periódico en su versión electrónica. También se resalta este papel de la Red como fuente de noticias en el estudio "Mediascope Europe 2007" que realiza la European Interactive Advertising Association (EIAA), en donde se especifica que las webs más visitadas por los internautas europeos son las de noticias, por el $65 \%$ de ellos, y en segundo lugar las de información local, por el $52 \%$. Esos porcentajes aumentan en el caso de los internautas españoles hasta el $80 \%$ y el $71 \%$, respectivamente.

En España, el número de personas de 14 y más años de edad que acceden a la prensa online está aumentado todos los años, pero también aumenta el porcentaje de individuos que acceden de forma conjunta tanto a la prensa online como a la impresa, es decir que leen ambos formatos a la vez (cuadro $n^{\circ} 1$ ). Hasta el año 2004, se han producido incrementos anuales en el número de lectores en todos los soportes. Pero a partir de dicho año se produce un cambio en las distintas, por una parte el número de lectores de la versión en papel de los periódicos disminuyen año a año, hasta reducirse en el año 2007 a un porcentaje de acceso del $36,0 \%$ entre los individuos de 14 o más años. Y por el contrario, aumenta tanto el número de lectores que solo acceden a la versión online de los periódicos, hasta un porcentaje del 2,0\%, como los lectores que acceden a las dos versiones de los periódicos, en papel y online hasta el 3,8\%. (San Pedro, Plaza y Restra, 2007:239). Entre los años 2004 y 2007, los periódicos en papel han sufrido una pérdida de consumo de 2,7 puntos porcentuales, que se reduce a 1,4 puntos, si a al porcentaje de individuos que solo leen prensa en papel, se les suma el correspondiente de los individuos que leen las dos versiones. Por su parte, en dicho periodo, el porcentaje de las personas que solo leen la prensa online, se ha duplicado, pasando del $1 \%$ al $2 \%$. Y el porcentaje de las personas 
que leen las dos versiones ha aumentado 1,3 puntos porcentuales, desde el $2,5 \%$ en el año 2004, hasta el 3,8\% en el 2007.

Cuadro 1. Grado de penetración de la prensa en papel, prensa online, y prensa en papel+online (porcentajes sobre individuos de 14 años y más).

\begin{tabular}{|l|l|l|l|l|}
\hline & $\begin{array}{l}\text { Sólo prensa en } \\
\text { papel (A) }\end{array}$ & $\begin{array}{l}\text { Sólo prensa } \\
\text { online (B) }\end{array}$ & $\begin{array}{l}\text { Prensa en papel } \\
\text { +prensa online(C) }\end{array}$ & $(\mathrm{A})+(\mathrm{C})$ \\
\hline 2000 & 35,8 & 0,2 & 0,5 & 36,3 \\
\hline 2001 & 35,1 & 0,4 & 0,8 & 35,9 \\
\hline 2002 & 36,1 & 0,8 & 1,4 & 37,5 \\
\hline 2003 & 38,0 & 0,8 & 1,8 & 39,8 \\
\hline 2004 & 38,7 & 1,0 & 2,5 & 41,2 \\
\hline 2005 & 38,3 & 1,2 & 2,8 & 41,1 \\
\hline 2006 & 36,9 & 1,5 & 3,3 & 40,2 \\
\hline 2007 & 36,0 & 2,0 & 3,8 & 39,8 \\
\hline
\end{tabular}

Fuente: AIMC, EGM Octubre de 2005 a Mayo de 2006 (2a Ola, 2006). Cuadro extraído de San Pedro, Plaza y Restra, La Eficacia Publicitaria de los Periódicos on-line y elaboración propia.

Dada la importancia de una correcta cuantificación del número de lectores de los periódicos en los dos soportes, se amplia la información sobre el tema con los datos que suministra el estudio de la Fundación del BBVA anteriormente mencionada. Según dicho estudio, el $41,5 \%$ de los encuestados sólo lee la edición impresa del periódico, el $10 \%$ sólo lee la edición digital del periódico, mientras que el $26,9 \%$ lee de forma habitual tanto la edición digital como la impresa. Por otra parte, el $19,0 \%$ de los encuestados no lee de forma habitual ningún tipo de periódicos, y el $2,6 \%$ no ha contestado a la pregunta. Según estos datos se puede estimar que la edición online actúa como bien sustitutivo de la edición impresa en el $10 \%$ de los encuestados, en aquellas personas que especifican que sólo leen la edición digital. Pero habría que partir del supuesto, que es un tanto discutible, de que estos lectores accederían a la versión en papel del periódico si no existiese la online. Por otra parte, esta información que proporciona el estudio de la Fundación BBVA se basa en la población de la muestra que ha utilizado Internet en los últimos tres meses.

De los encuestados que leen las dos versiones de los periódicos, el $68 \%$, leen la versión en papel de un periódico y la digital de otro. Y el $27 \%$, leen las dos versiones del mismo periódico. La principal razón que señalan los encuestados para leer los periódicos en los dos soportes es la de poder acceder a 
26 La demanda de los periódicos impresos y online: un análisis económico

información actualizada, que la especifican el 57\%. Otras razones relevantes son: poder contrastar la información en otro periódicos para el $37 \%$ y acceder a contenidos y servicios que solo están disponibles en Internet, el $27 \%$. En el caso de los lectores que contrastan la información leída en un periódico en papel, con las versiones online de otros periódicos, se puede considerar que estas versiones online actúan como bienes sustitutivos de sus ediciones correspondientes impresas, aunque también sería discutible el número total de ellos que comprarían más de un periódico en papel para poder contrastar las noticias.

Con el paso del tiempo aumenta el número de internautas que prefieren leer el periódico en Internet. Se ha elevado desde del $14,6 \%$ en el año 2005 , hasta el $21,2 \%$, en el 2008 , y lógicamente se reduce el porcentaje de los que prefieren el soporte de papel, del $75,7 \%$ al $63,4 \%$, respectivamente. Curiosamente las razones señaladas por los lectores para preferir la lectura en uno u otro soporte son muy parecidas, tanto en la estudio del año 2005, como en el del 2008. Las principales ventajas del soporte papel son: la facilidad de la lectura, según el $47,4 \%$, se puede leer vaya donde vaya el lector, un $32,7 \%$, no tener acceso en todas partes a un ordenador, indicado por el $10,5 \%$ y poder tener una copia en papel de lo que lee $5,3 \%$. Si se comparan estas razones, con las aducidas en el año 2005 , se observa que los porcentajes son muy parecidos, $46,2 \%, 34,0 \%$ y $9,1 \%$ respectivamente, para las tres primeras razones mencionadas anteriormente. Pero la posibilidad de poder guardar una copia en papel, no se mencionaba en dicho año. Por lo tanto, para los lectores del periódico impreso, se mantienen a lo largo del tiempo las ventajas especificadas de este soporte.

Por otra parte, los principales motivos por los que se prefiere leer la versión online del periódico son: la facilidad de encontrar la información que les interesa, para el $37,8 \%$, y que es un producto gratis: $28,0 \%$. Otras razones especificadas son: la facilidad para guardar las noticias $(9,2 \%)$, la información se actualiza constantemente $(7,5 \%)$, mejor calidad de las imágenes $(6,7 \%)$ y que pueden leer el periódico en cualquier momento $(4,8 \%)$. Si se compara estos motivos con los indicados en el año 2005, en el que la facilidad de encontrar la información es indicada por el $61,1 \%$, la gratuidad del producto por el $18,4 \%$, y la facilidad para guardar las noticias por el $7,1 \%$, se observa en dicha comparación que la gratuidad del periódico online es el único motivo que adquiere una mayor importancia. Por lo tanto, los criterios de preferencia de los lectores en los dos soportes son muy estables, y se mantienen a lo largo del tiempo. Además, en algunos casos, los lectores de cada uno de los soportes asignan el mismo tipo de ventaja comparativa al periódico impreso y al digital.

Únicamente el 7,4\% de los internautas que han accedido a la Red en los últimos tres meses de la muestra del estudio de la Fundación BBVA, han pagado por bajar un artículo. Por otra parte, el $6,2 \%$ no ha pagado pero estaría dispuesto a pagar por ello, pero el $80,9 \%$ de los internautas no ha pagado ni está dispuesto a 
pagar. El número de personas que no está dispuesto a pagar por leer un artículo es más elevado que aquellos que no están dispuestos a pagar por bajarse una película, música o software de la Red, es decir, la predisposición a pagar por los periódicos online es muy reducida. Sólo es menor la predisposición a pagar por acceder al buscador que se utiliza de forma habitual o por jugar en la Red. Claramente, en España el lector de periódicos online lo percibe como un producto gratuito, y es una costumbre muy establecida, no pagar por su consumo, por lo tanto, considera el lector que el producto se debe financiar exclusivamente por publicidad.

Los internautas, cuando buscan información y noticias en Internet, no acceden únicamente a los periódicos, sino también a las páginas de las principales cadenas de televisión y de radio y a los buscadores y otros proveedores online de noticias. De hecho según el EGM, del período año móvil febrero a noviembre de 2007, en el ranking que realiza la AIMC de los sitios de Internet con un mayor número de visitantes únicos en los últimos treinta días, la primera posición la ocupa el periódico Marca. El País ocupa la tercera posición, y la quinta As. Pero la web de la cadena de televisión Telecinco ocupa la sexta posición, y la web de Cuatro, tiene la décima posición. Por su parte, el séptimo y el décimo lo ocupan lugar los sitios de Internet de dos cadenas de radio: la Ser y Los 40 , respectivamente. Las restantes diez primeras posiciones no están ocupadas por medios: Terra tiene el segundo lugar, Páginas Amarillas el cuarto y Ya.com, el octavo. Este ranking refleja el elevado grado de competencia de los distintos medios en Internet, no solo entre ellos, sino que también tienen que competir con otros proveedores de información existentes en la Red, de hecho se considera que la mayor competencia para los periódicos online proviene de los sitios de buscadores y de las cadenas de televisión (Chaves et al., 2007: 248).

Un ejemplo de la competencia entre los distintos proveedores de noticias existente en Internet se puede encontrar en el estudio de ComScore titulado "Younger, Heavy Online News Consumers are Not Newspaper Readers, According to comScore Plan Metrix", del año 2008, en el que se analiza el acceso a los distintos medios online disponibles así como el grado de lectura de periódicos en papel y online según la edad del usuario, en el mercado norteamericano. En este estudio se demuestra tanto el alejamiento de los lectores de menor edad de los periódicos impresos, como que la edad del usuario marca el acceso a los distintos medios. También queda reflejado en dicho estudio como las personas que no leen de forma diaria periódicos impresos, visitan más sitios de noticias online que los que si los leen.

En el estudio de ComScore se clasifica a los lectores de los periódicos en papel según su frecuencia semanal de lectura en cuatro grupos: los "heavy newspaper readers" que los leen con una frecuencia de seis días por semana; los "medium newspapre readers", cuando la frecuencia semanal es de 3 a 5 días; los "light newspaper readers" si la lectura es de 1 o 2 veces semanales y 
28 La demanda de los periódicos impresos y online: un análisis económico

por ultimo los "non newspaper reader" que son las personas que no leen ningún periódico en papel durante la semana.

Y a continuación se asigna la probabilidad que tiene una persona determinada de pertenecer a uno de estos cuatro grupos en función de su edad. Afirma ComScore que los lectores de periódicos en papel pertenecen a los mayores tramos de edad: los lectores que tiene 65 o más años, tiene una probabilidad tres veces superior a la media, es decir son tres veces más propensos que la media de ser "heavy newspaper readers", siendo la probabilidad el doble de la media en los individuos con edades comprendidas entre 55 y 64 años. Pero esta probabilidad se reduce hasta un poco más que el valor de la media en los que tienen una edad comprendida entre 45 y 54 años. Por el contrario, en los que tienen de 18 a 24 la probabilidad de encontrarse entre este grupo de lectores es sólo un tercio de la media, aumentando esta probabilidad hasta la mitad del valor medio en los de 24 a 35 años. De forma análoga, los individuos más jóvenes, cuyo rango de edad está comprendido entre 18 y 24 años, tienen un 38\% más de probabilidad que la media de las personas de no leer a lo largo de la semana ningún periódico en papel, y esta probabilidad es un $27 \%$ más que la media en los individuos de 25 a 34 años. Claramente la edad de la persona marca su frecuencia de lectura semanal de periódicos.

Pero estos datos no quieren decir que las personas más jóvenes no estén interesadas en las noticias, y que no sean consumidoras de ellas porque no lean periódicos físicos, sino que tienen un modelo distinto de acceso a la información y visitan con una mayor frecuencia los sitios online de noticias. De hecho, según los resultados de este estudio de ComScore tienen una elevada propensión a visitar los sitios de noticias online y consumen más noticias online que la media de los individuos pero se informan en las web de los periódicos impresos y de las cadenas de televisión, o en sitios específicos de la Red. También se especifica en este estudio que los sitios online de periódicos de prestigio como The New York Times, The Chicago Tribune y LA Times tienen un elevado número de visitas que las realizan tanto los lectores de periódicos físicos, como aquellos que no compran sus ediciones en papel. De hecho, los porcentajes de visitas de los lectores clasificados como "heavy newspaper" son muy parecidos a los calificados como "non newspaper". Pero estos últimos tienen unos porcentajes de visitas algo mayores en los sitios online de noticias de las cadenas de televisión como CNN, Fox, CBS, ABC. Y en los sitios de noticias específicos online de Geoogle News Search, AOL, Yahoo y Digg, los porcentajes de visita de los "non newspaper" son también superiores que los de los "heavy newspaper".

Por otra parte, entre los "medium" y "light newspaper readers", también se produce un mayor porcentaje de visitas a los sitios de noticias online de las televisiones y propios de Internet, que a las web de los periódicos de prestigio. Por lo tanto, en el mercado norteamericano ya se está produciendo un cambio de las pautas de consumo de las noticias. Es previsible que en la medida en que 
se generalice el uso de Internet y de las conexiones de ADSL en los hogares españoles también se vaya produciendo un cierto cambio en las pautas de consumo de las noticias, aunque es difícil de prever si el cambio tendrá las mismas dimensiones. Además, desde la Comisión Europea se está intentando potenciar la generación de contenidos online por los medios y en la medida en que tenga éxito está iniciativa, lógicamente aumentará el consumo online de los mismo. Por otra parte, es necesario tener presente la importancia que en el mercado español ha tenido el consumo de los periódicos gratuitos impresos, y ésta es una variable que también hay que tenerla en cuenta.

Lógicamente el grado de desarrollo de la Sociedad de la Información de un país es una variable básica que hay que considerar cuando se habla del consumo de los medios online por parte de sus ciudadanos. Aunque los incrementos en el número de internautas que se han producido en España en los últimos años están provocando una similitud progresiva entre la composición demográfica de los internautas y de la población total (Observatorio Red.es, 2008: 23). El usuario medio de Internet español es un hombre, con un nivel de formación de estudios universitarios o bien de segunda etapa de educación secundaria y en una situación laboral de activo ocupado (Observatorio Red.es, 2008: 3). Esta realidad se especifica en los distintos informes sobre Internet en España, por ejemplo en el estudio de la Fundación BBVA anteriormente mencionado se indica que en el perfil de los usuarios españoles destacan los hombres, jóvenes, personas con un nivel socioeconómico medio o medio-alto y que tienen un mayor nivel de formación. También se resalta en dicho estudio las fuertes diferencias que se producen dentro del perfil del usuario de la Red español por estudios y clase social y el elevado salto digital existente entre las distintas generaciones. Por otra parte, las diferencias de género revisten una menor amplitud (Fundación BBVA, 2008: 6).

Por todo ello, el análisis de los lectores de los periódicos impresos y online se va a subdividir en dos grandes apartados. En primer lugar se analizan sus principales características demográficas, y a continuación las socioeconómicas. La exposición se centrará en el consumo de ambos medios y en el perfil de su audiencia según los datos que proporcionan las publicaciones de la AIMC, EGM Prensa 3er año móvil 2007 y EGM $3^{\text {a }}$ Ola (Febrero a Noviembre 2007). La exposición se completa con el análisis de las principales características de los internautas españoles también utilizando como fuente a la AIMC, y su comparación con las características de los lectores de periódicos online. A lo largo del artículo se compararán los datos del año 2007 con los de años anteriores para ver la evolución de los lectores utilizando para ello el trabajo de Juan Manuel de Toro, Visitación Lozano y Belén Milan, "La prensa digital", cuarta parte del Libro Blanco de la Prensa Diaria 2008, de la Asociación de Editores de Diarios Españoles (AEDE). También se utilizará esta publicación como fuente de información de los periódicos impresos. Estas fuentes de información básicas se contrastarán con los datos de otros informes nacionales y extranjeros. 


\section{PRINCIPALES CARACTERÍSTICAS DESDE EL PUNTO DE VISTA DEMO- GRAFICO DE LOS LECTORES DE PERIÓDICOS}

En los periódicos online se acentúan las diferencias de género que se producen en los periódicos impresos, con un grado de consumo muy superior en los hombres, 9,47 de cada 100, mientras que en las mujeres es del 3,24. Es decir, el nivel de su consumo en los hombres es casi tres veces mayor que en las mujeres. $Y$ es una diferencia mucho mayor que la que se produce en el caso de los periódicos impresos, con un consumo de 50,55 de cada 100 hombres y de 32,49 en las mujeres. También esta brecha es mucho mayor que la que se produce en el grado de penetración de Internet en la población española según género, con 31,1 de cada 100 hombres y 21,6 de cada 100 mujeres. Por lo tanto, el menor número de lectoras online de periódicos no se justifica sólo por un menor grado de acceso de las mujeres a Internet, donde las diferencias existentes son menores, sino que también tiene que haber otras causas que lo motiven. Este bajo grado de consumo femenino lleva a que en el perfil de la audiencia de los periódicos online los hombres supongan el $73,67 \%$ de los lectores y las mujeres sólo el $26,33 \%$. Por lo tanto, se agravan las distancias de género que existen en los periódicos impresos, donde las mujeres son el 40,19\% de los lectores. En Internet también se produce un peso más igualitario que en los periódicos online, siendo las mujeres el $42,0 \%$ de los usuarios.

Si se compara la situación actual de la prensa online con la que existía hace unos años, se observa un retroceso con respecto a la situación que había en el año 2006. En dicho año el peso en términos porcentuales de las mujeres era del $35,2 \%$, es decir la situación actual se aproxima a la existente en el año 2005 en donde el peso de las mujeres era de sólo el $21,1 \%$, o en el año 2002 , con un porcentaje del $29,6 \%$. Si se considera el análisis los últimos seis años, y con la excepción de los tres mencionados, en todos los demás años el peso que representan las mujeres en el total de la población lectora de periódicos online siempre ha estado por encima del 30\% (Toro et al., 2007: 146).

En la comparación de la edad de los usuarios de los periódicos en sus dos versiones y de Internet se aprecian tendencias diferentes. El mayor consumo de periódicos impresos se produce en los individuos más maduros y en los de edades centrales, con un máximo de 48,74 lectores de cada 100 individuos de 45 a 54 años. El segundo colectivo con mayor consumo lo forman las personas con una edad comprendida entre los 35 y los 44 años, con un grado de penetración de la prensa impresa del $46,53 \%$. Por otra parte, los niveles más reducidos de consumo de periódicos en papel se producen entre los más jóvenes, con 31,70 lectores de cada 100 individuos entre 14 a 19 años y en las personas de mayor edad, con 27,94 lectores de cada 100 individuos de 65 y más años (cuadro $n^{\circ} 2$ ). 
Cuadro 2. Grado de penetración (por edad).

\begin{tabular}{|l|l|l|l|}
\hline & Periódicos impresos & Periódicos online & Internet \\
\hline 14 a 19 & 31,70 & 7,15 & 54,4 \\
\hline 20 a 24 & 44,00 & 11,36 & 51,1 \\
\hline 25 a 34 & 45,58 & 10,46 & 39,3 \\
\hline 35 a 44 & 46,53 & 7,71 & 30,0 \\
\hline 45 a 54 & 48,74 & 6,11 & 23,3 \\
\hline 55 a 64 & 42,65 & 3,47 & 11,9 \\
\hline 65 y + & 27,94 & 0,61 & 2,0 \\
\hline
\end{tabular}

Fuente: AIMC, EGM Prensa, 3er Año Móvil 2007, EGM $3^{\text {a }}$ Ola (Febrero a Noviembre 2007).

El alejamiento de los jóvenes de los periódicos impresos es un fenómeno que se produce en diferentes países. Por ejemplo, el informe realizado por Merrill Brown "Abandoning the News", y publicado por la Carnegie Corporation of New York, es un informe clásico sobre esta temática. En él se realiza un amplio análisis del consumo de noticias por parte de los jóvenes norteamericanos de 18 a 34 años, y se resalta el cambio en los hábitos de consumo de noticias que se está produciendo en este colectivo, ya que sus conocimientos de las nuevas tecnologías les llevan a dejar de consumir los medios tradicionales y los sustituyen por otras fuentes de información, como portales, blogs, agregadores de noticias. Además se especifica que es un cambio de tendencia que llevará al abandono en el futuro del consumo de los medios tradicionales, lo que implica que el público más joven se aleja de forma irreversible de las fuentes tradicionales de las noticias ya que se considera en dicho estudio que el aumento de edad de estos jóvenes no alterará sus pautas de consumo de los medios (Brown, 2005: 1-2, 5).

En otros informes internacionales, también se resaltan las mismas tendencias en el consumo de noticias por parte de la población más joven, por ejemplo, en el estudio de la Asociación Mundial de Periódicos (WAN), "Youth Media DNA", presentado en el Congreso Mundial de Periódicos celebrado en Ciudad del Cabo, Sudáfrica, en el año 2007. En él se detallan las principales tendencias en el consumo de medios de 100 jóvenes de 15 a 29 años de diferentes países, entre ellos en España. En este estudio se especifica cómo los jóvenes utilizan distintas fuentes de información, también se indica cómo el uso de los nuevos medios resta tiempo en los jóvenes al consumo de los medios tradicionales. En este estudio de la WAN también se incide en las distintas pautas de consumo de noticias por parte de la población más joven, con la creciente importancia que tienen las redes sociales como fuente de información. Otros temas que se analizan son la desconfianza de los jóvenes en utilizar información de una sola fuente de noticias, la consideración de que las informaciones de los medios tradicionales son más 
32 La demanda de los periódicos impresos y online: un análisis económico

fiables, precisas y fidedignas que las de los nuevos medios. Este estudio de la WAN es preliminar a un estudio más amplio en el que participarán 1000 jóvenes, lo que permitirá tener una amplia visión del consumo de medios de los jóvenes en diferentes países y dará pautas a la industria de los periódicos para intentar recuperarlos como lectores.

En relación al mercado español, se considera que los principales motivos por los cuales se produce un alejamiento del público más joven de los periódicos impresos son la falta de convivencia con los periódicos en sus hogares, la falta de adecuación de los contenidos de la prensa para los jóvenes y la influencia de las nuevas tecnologías (Arroyo, 2007: 280). Además, los jóvenes españoles consideran que las noticias políticas tienen demasiado peso en los periódicos impresos y sus contenidos se centran en un grado mucho menor en los temas que más les interesan a ellos, como por ejemplo la realidad social próxima, fundamentalmente la de índole social y deportiva, y las noticias vinculadas con la comunidad en la que viven. Precisamente como la prensa gratuita se centra en un grado mayor en dichos temas que la prensa escrita es por lo que tiene más éxito entre el público más joven (Sabés, 2005: 102). También en el apartado referido a España del estudio de la WAN anteriormente mencionado se resaltan las preferencias por las noticias locales de los jóvenes españoles (WAN, 2007: 14).

El consumo de Internet en España sigue una tendencia contraria a la que tiene el consumo de los periódicos impresos, pues sus mayores niveles de consumo se producen entre los más jóvenes, 54,4 personas de cada 100 entre 14 y 19 años son usuarios de la Red y 51,1 individuos de cada 100 entre 20 y 24 años. A partir de esas edades se reduce el consumo de forma muy acusada (cuadro $n^{\circ} 2$ ). Por su parte, el mayor grado de consumo de periódicos online se produce entre los 20-24 años con 11,36 lectores de cada 100 personas de dicha edad y en los que tienen de 25 a 34 años con 10,46 lectores. Por el contrario en la prensa offline, estos tramos de edad ocupan la cuarta y tercera posición, respectivamente, entre el colectivo de lectores. También es elevado el nivel de consumo de periódicos online, en términos relativos, entre los colectivos de 35 a 44 años con 7,71 lectores por cada 100 personas de dicha edad y en los que tienen de 14 a 19 años con 7,17 lectores (cuadro $n^{\circ} 2$ ).

Por el contrario, entre el colectivo de 45 a 54 años, que son el principal grupo lector de periódicos impresos, con un nivel de consumo del $48,74 \%$, en la versión online su consumo se reduce a sólo el $6,11 \%$, ocupando la quinta posición en el colectivo de lectores. Obviamente, dicho colectivo también tiene un bajo grado de consumo de Internet, el 23,3\%, pero es un nivel de consumo muy superior al de los periódicos online. En los colectivos de mayor edad el consumo de periódicos online se reduce sensiblemente hasta sólo 0,61 lectores entre 100 personas de 65 y más años de edad. Por lo tanto, se produce en España un cierto acercamiento de los jóvenes a los periódicos, en su versión online, comparado con los bajos índices de lectura de la versión impresa. 
Los reducidos niveles de consumo comentados unidos a los cambios demográficos producidos en España en los últimos años conllevan a que en el análisis del perfil de la audiencia, considerando como variable la edad del lector, las personas con una edad comprendida entre 35 y 44 años sean el principal colectivo lector en el caso de los periódicos impresos y representen el 21,81 de cada 100 lectores. El segundo colectivo que más periódicos lee lo integran las personas que tienen entre 25 y 34 años con el $21,10 \%$ y el tercero, con el $18,09 \%$, los que tienen entre 45 y 54 años (cuadro $n^{\circ} 3$ ). Por otra parte, los colectivos que tienen un menor peso entre los lectores de periódicos impresos son las personas más jóvenes. Además, es necesario tener en cuenta en este análisis que dentro de los periódicos en papel están también incluidos los periódicos gratuitos, que tienen una elevada demanda entre el público de menor edad. Este alejamiento de los lectores más jóvenes de los periódicos impresos implica un continuo incremento de la edad promedio del lector de prensa en papel, que se ha elevado desde los 43,2 años en el año 2005, hasta los 43,5 años en el 2006 (Toro et al., 2007: 35).

El peso de los diferentes colectivos varía de una forma sensible con respecto a la situación que se acaba de comentar en el perfil de los periódicos online. En este caso la primera posición la ocupan las personas que tienen una edad comprendida entre 25 y 34 años con el $31,85 \%$, es decir, que de cada 100 lectores de periódicos online, 31,85 son individuos que tienen dicha edad, y la segunda los que tienen entre 35 y 44 años, con el $23,77 \%$. La tercera posición es ocupada, como en el caso de los periódicos impresos, por las personas de 45 a 54 años, con el $14,92 \%$. Pero se produce un cambio sustancial en las siguientes posiciones, ocupadas por los individuos más jóvenes. Por otra parte, es significativo el reducido peso que tienen los lectores de 65 y más años, pues los lectores de periódicos online de dicha edad representan sólo el 1,89\% del total de sus lectores (cuadro $n^{\circ} 3$ ).

Cuadro 3. Perfil de la audiencia (por edad).

\begin{tabular}{|l|l|l|l|l|}
\hline & $\begin{array}{l}\text { Periódicos } \\
\text { impresos }\end{array}$ & $\begin{array}{l}\text { Periódicos } \\
\text { online }\end{array}$ & Internet & $\begin{array}{l}\text { Intensidad acceso } \\
\text { Periódicos online }\end{array}$ \\
\hline 14 a 19 & 5,52 & 8,2 & 14,9 & 55,0 \\
\hline 20 a 24 & 7,35 & 12,48 & 13,4 & 93,1 \\
\hline 25 a 34 & 21,10 & 31,85 & 28,6 & 111,4 \\
\hline 35 a 44 & 21,81 & 23,77 & 22,2 & 107,1 \\
\hline 45 a 54 & 18,09 & 14,92 & 13,6 & 107,7 \\
\hline 55 a 64 & 12,88 & 6,9 & 5,7 & 121,1 \\
\hline 65 y + & 13,25 & 1,89 & 1,5 & 126 \\
\hline
\end{tabular}

Fuente: AIMC, EGM Prensa, 3er Año Móvil 2007, EGM $3^{\text {a }}$ Ola (Febrero a Noviembre 2007) y elaboración propia. 
34 La demanda de los periódicos impresos y online: un análisis económico

Si se comparan estos datos del año 2007 con las cifras de años anteriores, se puede aprecia cómo ha disminuido, medido en términos porcentuales, el peso de los lectores de 20 a 24 años, y por el contrario ha aumentado el peso de los lectores que tienen 45 y más años (Toro et al., 2007: 147). Estos cambios probablemente se deban al envejecimiento del perfil medio del usuario de Internet que ha habido en España en los últimos años, toda vez que se está produciendo un uso más generalizado de la Red por parte de la población española.

Por su parte, en el perfil de la audiencia de Internet, las primeras posiciones las ocupa el grupo de personas que tiene una edad comprendida entre los 25 y 34 años con el 28,6\% del total de usuarios de la Red, y los de 35 a 44 años, con el $22,2 \%$. En las últimas posiciones, lo que refleja un menor grado de utilización de Internet en términos relativos, se encuentran las personas de más edad (cuadro $n^{\circ} 3$ ). Claramente, todavía el internauta español sigue teniendo un perfil propio y con diferencias sensibles en relación a las audiencias de otros medios (Camino, Marín y Armentia, 2006).

Otra forma de analizar el nivel de lectura de los periódicos online en los diferentes tramos de edad consiste en comparar el porcentaje de usuarios de Internet de cada uno de dichos tramos con su correspondiente porcentaje de lectores de periódicos online. Es decir, sería comparar el porcentaje que de cada 100 internautas representan las personas de un determinado grupo de edad, con el porcentaje que dicho grupo representa de cada 100 lectores de periódicos online. Por lo tanto, es una aproximación a la cuestión de que, una vez que han accedido a Internet, cuántos individuos de cada una de las diferentes edades leen periódicos. Y para facilitar el análisis se calcula un indicador que muestra cuánto supone en términos porcentuales el número de lectores de cada tramo de edad en relación a su número de internautas. Sería un indicador del consumo, en términos relativos, de periódicos online de cada uno de los diferentes tramos de edad.

A partir los 25 años de edad es mayor el porcentaje de lectores de cada una de las edades, en el total de lectores de periódicos online, que el valor que representa el porcentaje de internautas de esa edad en el total de usuarios de Internet. Por lo tanto, una vez que han accedido a Internet, es a partir de los individuos con una edad mayor de 25 años donde sería más probable encontrar a los lectores de periódicos online en España.

Además, aparecen unos resultados en el indicador un tanto sorprendentes: los mayores niveles de lectores, en términos relativos, se producen en los individuos de más edad, con unos porcentajes de lectura de periódicos online muy elevados, concretamente el $126 \%$ en las personas que tienen 65 y más años y el $121,1 \%$ en el tramo de edad anterior, de 55 a 64 años. Es decir, los individuos de dichos colectivos que disponen de Internet acceden en un mayor grado a los periódicos online que las personas de los otros tramos de edad, ya que su grado de uso de los periódicos online es mayor que su nivel de utilización de la Red, todo ello en términos relativos sobre el total de la población. En tercer lugar, según 
los resultados del indicador, se sitúan los individuos que tienen entre 25 y 34 años, lo que es una situación que era inicialmente previsible, con $111,4 \%$, y a continuación los tramos de 45 a 54 años y de 35 a 44 años, con el 107,7\% y $107,1 \%$ respectivamente (cuadro $n^{\circ} 3$ ). Por su parte, las personas más jóvenes tienen unos valores sensiblemente más pequeños, y por debajo de 100 , los de 14 a 19 años, el $55,0 \%$ y los de 20 a 24 , el $93,1 \%$, datos que reflejan un bajo grado de uso de los periódicos online, en términos relativos, en estas edades, y que es menor el consumo de periódicos online que su nivel de consumo de Internet. Estos resultados implican que en la medida en que se produzca en España un mayor uso de la Red por las poblaciones de más edad, previsiblemente aumentará en una cuantía mayor el número de lectores de periódicos online.

Estos bajos resultados de lectura de los periódicos online, en términos relativos, por parte de la población más joven, concuerdan con los resultados del estudio de la WAN anteriormente mencionado, donde muchos de los jóvenes españoles entrevistados indican que no leen frecuentemente periódicos online y que prefieren leer periódicos impresos a leerlos en pantalla (WAN, 2007: 14).

\section{PRINCIPALES CARACTERÍSTICAS DESDE EL PUNTO DE VISTA DE LA CLASE SOCIAL DE LOS LECTORES DE PERIÓDICOS}

Según los datos del EGM, el nivel de consumo de los periódicos impresos está fuertemente relacionado con la clase social del lector, además su consumo se reduce a medida que desciende la clase social del lector. La AIMC estima la clase social de los encuestados en función del nivel de estudios y de la ocupación del sustentador principal de la familia. En la clase alta el consumo de periódicos impresos es de 62,15 lectores de cada 100 personas de dicha clase, que es una cifra cuatro veces superior que en la clase baja, con solo 15,25 lectores. Además, el nivel de consumo de la clase alta es el doble que en la media-baja, donde los periódicos tienen un grado de penetración del 31,45\% (cuadro $n^{\circ} 4$ ).

Estas diferencias de consumo entre las diferentes clases sociales se agudizan en el caso de Internet, en donde el número de usuarios de clase alta, 59 de cada 100, es más de doce veces el número de usuarios en hay en la clase baja, que sólo es de 4,8, y representa casi cuatro veces más que el valor de la clase media-baja, que tiene un nivel de consumo de Internet de 14,2 personas de cada 100. Por otra parte, el consumo de la Red en la clase media baja es tres veces mayor que en la clase baja (cuadro $n^{\circ} 4$ ). Por lo tanto, existe en España una fuerte brecha digital entre las diferentes clases sociales. Además, está brecha entre clases es mayor en el uso de Internet que en la lectura de periódicos impresos. Aunque en los últimos años se está produciendo un mayor acceso de todas las clases a la Red, se considera que por ahora Internet es un ámbito más elitista que la prensa escrita (Caminos, Marín, y Armentia, 2006). 
36 La demanda de los periódicos impresos y online: un análisis económico

Cuadro 4. Grado de penetración (por clase social).

\begin{tabular}{|l|l|l|l|}
\hline & Periódicos impresos & Periódicos online & Internet \\
\hline Alta & 62,15 & 17,85 & 59,0 \\
\hline Media-alta & 53,82 & 11,15 & 42,7 \\
\hline Media-media & 44,19 & 6,32 & 28,6 \\
\hline Media-baja & 31,45 & 2,99 & 14,2 \\
\hline Baja & 15,25 & 0,54 & 4,8 \\
\hline
\end{tabular}

Fuente: AIMC, EGM Prensa, 3er Año Móvil 2007, EGM $3^{\text {a }}$ Ola (Febrero a Noviembre 2007).

Todavía aparecen unas diferencias mayores entre las distintas clases sociales en el consumo de los periódicos online. En este caso el grado de consumo entre las personas de clase alta, con 17,85 lectores de cada 100, es más de treinta y tres veces mayor que en la clase baja, que sólo tiene un consumo de 0,54 lectores y cerca de seis veces el consumo de la clase media-baja (cuadro $n^{\circ} 4$ ). Claramente las nuevas tecnologías no facilitan un mayor acceso de las clases menos favorecidas a los periódicos.

Además en el caso de los periódicos online se agravan las diferencias existentes no sólo entre los extremos de las clases sino entre todas ellas. Por ejemplo, en los periódicos en papel el nivel de consumo de la clase media-media es un $71 \%$ del consumo de la clase alta. En cambio, en los periódicos online este porcentaje se reduce hasta el $35,41 \%$. Y esta diferencia no se produce sólo por un menor grado de acceso de los usuarios de esta clase a Internet, ya que su nivel de uso de la Red es el $48,47 \%$ del que tiene la clase alta; obviamente tampoco obedece al precio del producto, ya que el acceso a los periódicos online es gratuito para la mayoría de los contenidos, y en mucho casos para todo el periódico.

En el análisis del perfil de la audiencia de los medios analizados, se puede apreciar en primer lugar que el porcentaje más elevado de lectores pertenece a la clase media, con valores del $43,96 \%$ en el caso de los periódicos en papel, y del $40,48 \%$ en los online. También aumenta el peso, en términos relativos, de las clases más favorecidas en el cado de los periódicos online, con respecto a los impresos. De cada 100 lectores de prensa online, 20,31 son de clase alta y 23,45 son de clase media-alta, en cambio estos valores se reducen en la prensa física al 12,99 y 18,88 respectivamente. $Y$ en consonancia se reduce el peso, en términos porcentuales, de la clase media-baja del $21,29 \%$ al $15,09 \%$ y especialmente de la clase baja del $2,87 \%$ al $0,67 \%$ (cuadro $n^{\circ} 5$ ). Como se ha comentado al analizar el consumo que tienen las distintas clases, con las nuevas tecnologías se están alejando las clases menos favorecidas de los periódicos online, y se agrandan las diferencias existentes en los porcentajes de lectores según las distintas clases de la prensa física, que ya de por sí son muy elevados. 
Las diferencias de lectura de los periódicos online entre los individuos de las distintas clases no vienen motivadas sólo por un menor grado del uso de Internet entre ellas, ya que el nivel de lectura de los periódicos online de las clases menos favorecidas es mucho menor que su acceso a la Red. Sólo la clase alta y mediaalta tienen porcentajes mayores de lectores, $20,31 \%$ y $23,45 \%$ respectivamente, que de usuarios de Internet, $16,1 \%$ y $21,5 \%$, por ese orden. Las diferencias que se producen son especialmente significativas en la clase baja, ya que ostentan sólo 0,67 lectores de cada 100 lectores de periódicos online y 1,4 internautas de cada 100 usuarios de la Red (cuadro $n^{\circ} 5$ ). En el caso de dicha clase, su porcentaje en el total de lectores supone menos de la mitad que su porcentaje en el total de internautas.

Cuadro 5. Perfil de la audiencia (por clase social).

\begin{tabular}{|l|l|l|l|l|}
\hline & $\begin{array}{l}\text { Periódicos } \\
\text { impresos }\end{array}$ & $\begin{array}{l}\text { Periódicos } \\
\text { online }\end{array}$ & Internet & $\begin{array}{l}\text { Intensidad acceso } \\
\text { Periódicos online }\end{array}$ \\
\hline Alta & 12,99 & 20,31 & 16,1 & 126,15 \\
\hline Media-alta & 18,88 & 23,45 & 21,5 & 109,07 \\
\hline Media-media & 43,96 & 40,48 & 43,9 & 92,19 \\
\hline Media-baja & 21,29 & 15,09 & 17,1 & 88,25 \\
\hline Baja & 2,87 & 0,67 & 1,4 & 47,86 \\
\hline
\end{tabular}

Fuente: AIMC, EGM Prensa 3er Año Móvil 2007y EGM Febrero a Noviembre 2007 (3a Ola) y elaboración propia.

Para analizar de una forma más amplia el grado de uso de los periódicos online por parte de las distintas clases sociales, se va a relacionar el porcentaje que supone cada clase en el total de usuarios de Internet con el porcentaje que tiene esa misma clase en el total de lectores. De esta forma se elimina en el cálculo la barrera que supone disponer de acceso a Internet, y es una aproximación a la cuestión de una vez que han accedido a la Red, cuántas personas de cada clase leen periódicos online. Por lo tanto, sería un indicador del grado de uso, en términos relativos, de los periódicos online por parte de cada una de las diferentes clases sociales.

Los mayores valores del indicador corresponden a la clase alta y media alta, lo que refleja que el porcentaje de lectores de estas clases es mayor que su porcentaje en el total de usuarios de Internet, concretamente son mayores en un $126,15 \%$ y en un $109,07 \%$, respectivamente. Estos valores indican un alto grado de uso de los periódicos online por los usuarios de estas clases. En las demás clases los porcentajes son inferiores a 100, y es especialmente reducido el valor que corresponde a la clase baja, el $47,86 \%$, motivado porque el peso que esta 
38 La demanda de los periódicos impresos y online: un análisis económico

clase tiene en el total de usuarios de Internet, el 1,4\%, es mucho mayor que su peso en términos porcentuales, en los lectores de periódicos online, que es sólo el $0,67 \%$. Por su parte, el indicador tomas los valores de $92,19 \%$ y $88,25 \%$ respectivamente, en la clase media-media y en la clase media-baja (cuadro $n^{\circ} 5$ ), lo que refleja una menor diferencia entre sus respectivos porcentajes de usuarios de Internet y de lectores de periódicos online. Si estas relaciones de mantienen a lo largo del tiempo, un incremento del número de usuarios de Internet de las clases menos favorecidas implicará un incremento, aunque de una cuantía menor, en el número de lectores de los periódicos online.

\section{CONCLUSIONES}

El uso de las nuevas tecnologías ha potenciado el acceso a las noticias a través de esta vía. En este sentido se aprecia un incremento de lectura de periódicos online respecto a los impresos. Internet, de hecho permite acceder a un campo informativo muy amplio y conlleva otras ventajas respecto a las ediciones de prensa impresa. No se trata sólo del factor de la gratuidad, sino de la posibilidad para el usuario de una lectura simultánea y, por tanto, de cotejo informativo ofrecido por los diversos periódicos online.

El análisis de lectores desde el punto de vista demográfico y por estatus socioeconómico arroja una situación en la que el incremento de prensa online es evidente. Por género, en los periódicos online se acentúan las diferencias que se producen en los periódicos impresos, con un grado de consumo de ellos muy superior en los hombres que en las mujeres. Respecto a la edad, se evidencia un abandono de periódicos impresos por parte de los jóvenes y una tendencia al incremento de lectores online de edad media (de 45 y más años) por envejecimiento del perfil medio del usuario de Internet.

Asimismo, desde el punto de vista socioeconómico, se constata un alto grado de uso de periódicos online entre las clases alta y media alta. Aunque se espera un mayor aumento de usuarios de Internet en las clases menos favorecidas, sin embargo se cree que el número de lectores de periódicos online en estas clases sea siempre menor respecto a los demás estamentos.

\section{REFERENCIAS BIBLIOGRÁFICAS}

ASOCIACIÓN PARA LA INVESTIGACIÓN DE MEDIOS DE COMUNICACIÓN (2008): Navegantes en la Red. $10^{\circ}$ encuesta AIMC a usuarios de Internet. Madrid: AIMC.

- (2007a): Estudio General de Medios, Resumen General ( $3^{\circ}$ ola) Febrero a Noviembre de 2007. Madrid: AIMC.

- (2007b): EGM Prensa 3er Año Móvil 2007. Madrid: AIMC. 
ARROYO CABELLO, M.: "Los jóvenes y la prensa: hábitos de consumo y renovación de contenidos". Ámbitos, nº 15 (2006), pp. 271-282.

BROWN, M.: "Abandoning the News". Carnegie Reporter, vol. 3, n.2 (Spring 2005) New York: Carnegie Corporation of New York.

CARNEGIE CORPORATION (2005): Use Of Sources For News. New York: Carnegie Corporation of New York.

CAMINOS MARCET, J.M., MARÍN MURILLO, F. y ARMENTIA VIZUETE, J.I. (2006): "Las audiencias ante los cambios en el ciberperiodismo". Revista Latina de Comunicación Social (en línea) n 61 (2006). [Consulta: 8 de abril de 2008].

<http://www.ull.es/publicaciones/latina/200607Caminos.htm>

CHAVES SÁNCHEZ, L., PLAZA, P. y SAN PEDRO, M.: Retos y Oportunidades para la Prensa en Internet. En BEL MALLÉN, J. I. et al. (coord.) (2006): Libro Blanco de la prensa diaria. 2007. Madrid: Asociación de Editores de Diarios Españoles (AEDE), pp. 229-259.

COMSCORE (2008): Younger, Heavy Online News Consumers are Not Newspaper Readers, According to comScore Plan Metrix, press release 13 de marzo de 2008. Reston: ComScore.

EUROPEAN INTERACTIVE ADVERTISING ASSOCIATION (2007): Mediascope Europe 2007. Londres: European Interactive Advertising Association.

FUNDACIÓN BBVA (2008): Segundo Estudio sobre Internet en España. Madrid: Fundación BBVA, Departamento de Estudios Sociales.

- (2005): Estudio sobre Internet en España. Madrid: Fundación BBVA, Departamento de Estudios Sociales.

OBSERVATORIO RED.ES (2008): Perfil sociodemográfico de los internautas. Análisis de datos INE 2007. Observatorio Nacional de las Telecomunicaciones y de la Sociedad de la Información (ONTSI). Madrid: Red.es y Ministerio de Industria, Turismo y Comercio.

SABÉS TURMO, F.: “Jóvenes y medios: ¿encuentro o desencuentro?". Cuadernos de Periodistas, $\mathrm{n}^{\circ} 5$ (Diciembre 2005), pp. 101-108.

TORO MARTÍN de J.M., LOZANO MUÑOZ, V. y MILÁN LÓPEZ, M.: Datos generales sobre difusión y audiencia, La difusión controlada y la audiencia por diarios y La prensa digital. En BEL MALLÉN, J. I. et al (coord.) (2007): Libro Blanco de la prensa diaria. 2008. Madrid: Asociación de Editores de Diarios Españoles (AEDE), pp. 18-121 y 135-150.

WORLD ASSOCIATION OF NEWSPAPERS (2007): Youth Media DNA. Decoding youth as news \& information consumers Phase two report. París: WAN.

\section{Breve semblanza biográfica del autor}

Julio Larrañaga Rubio es Profesor del Departamento de Análisis Económico en la Facultad de Ciencias de la Información (UCM). Tiene distintas publicaciones sobre diversos aspectos económicos de los medios, y de la Sociedad de la Información. 